\title{
Robust Image Compression Algorithm using Discrete Fractional Cosine Transform
}

\author{
VIVEK ARYA \\ Department of Electronics \& Communication Engineering (FET) \\ Gurukula Kangri (Deemed to be University) Haridwar, INDIA
}

\begin{abstract}
The discrete fractional Fourier transform become paradigm in signal processing. This transform process the signal in joint time-frequency domain. The attractive and very important feature of DFrCT is an availability of extra degree of one free parameter that is provided by fractional orders and due to which optimization is possible. Less execution time and easy implementation are main advantages of proposed algorithm. The merit of effectiveness of proposed technique over existing technique is superior due to application of discrete fractional cosine transform by which higher compression ratio and PSNR are obtained without any artifacts in compressed images. The novelty of the proposed algorithm is no artifacts in compressed image along with good CR and PSNR. Compression ratio (CR) and peak signal to noise ratio (PSNR) are quality parameters for image compression with optimum fractional order.
\end{abstract}

Key-words:- Image compression, fractional transform, discrete fractional cosine transform, redundancy.

Received: March 5, 2021. Revised: October 10, 2021. Accepted: December 1, 2021. Published: January 5, 2022.

\section{Introduction}

The need for robust techniques that can store visual information and transmit has been increased in the advancement of multimedia applications [1]. For downloading the images from internet is a time consuming process and because of this, nowadays image compression become an attractive tool. Therefore, the requirement of efficient and effective algorithms that can provide high compression ratio with less information loss has increased [2]. Image data is the major portion of multimedia communication and it consumes more bandwidth [3] during transmission. Hence, development of optimum and novel techniques for image compression has become important paradigm [4]. Various lossy and lossless image compression algorithms have been developed for the increasing need of medical images, virtual conferencing and multimedia. The existing technique's model based on the analyzing two dimensional singularities and getting the captivating characteristics such as high peak-signal-to-noise ratio (PSNR). Discrete cosine transform (DCT) [5-8] is a very useful and important transform for compression and it is an adaptation of Fourier series. DCT provides fewer transform coefficients [9] after approximation of a signal. The Discrete sine transform (DST) is a factitive transform of DCT. Nowadays, DST is used in compression applications of audio coding and low rate image [1011]. Discrete Walsh Hadamard transform (DWHT) is the simplest transform among all, although its energy compaction capability very inferior than DCT. Therefore, it does not used for image or data compression [12-13]. DST, KLT and DCT are the linear orthogonal blocked transforms, which remove the correlating information or pixels inside the block. These transforms do not work well with interrelating over the block boundaries [14]. In last few years, hybrid fractal image compression techniques [15] are more in demand due to high compression rate while it needs more execution time because of high complexity.

In 1807, Jean Baptise Joseph Fourier introduced Fourier transform, while he was working with a heat conduction dilemma. However, with the advancement of research areas and theme, in 1929, the fractional power of Fourier transform noticed in the mathematical literature [16-18]. The Fractional Fourier Transform (FrFT) is commonly called as rotational or angular Fourier transform in different research papers [19-20]. Quantum mechanics [21], signal processing [22-24], pattern recognition [25], and optical, video and audio processing [26-27] are 
the significant applications of FrFT. In optical domain, the continuous FrFT is applied [28]. It is a scientifically and systematically proved fact that difference gives a richer solution set than their continuous limit differential equations [29]. In FrFT domain the decomposition of continuous discrete signals and systems has been developed [30]. Nowadays, FrFT have various authentic application areas [31-36,45]. In last few years, two dimensional discrete fractional cosine transform (DFrCT) is applied for pattern recognition, encryption and image compression [46] due to its robustness and reliability. Some other image compression algorithms also proposed by experts [47] where they have used discrete cosine transform. The important research gaps in the field of image compression are removal of correlating information from the image and obtained less PSNR and more blocking artifacts in compressed images. The main motivation for the research is to provide the robust image compression technique which work efficiently on all type of images and can give good compression ratio along with good PSNR.

\section{Proposed Image Compression Model}

Two dimensional discrete fractional cosine transform (DFrCT) is utilized at optimum value of fractional order. The quantization of all those coefficients is carried out to arbitrarily remove the coefficients that consist of very less information. This is achieved by adjusting the threshold value or cutoff value of the quantizer also called the coarseness. After this zigzag and Huffman coding is applied for further compression. For decompression of image, Huffman decoding and zigzag decoding was performed respectively. Now, inverse DFrCT (IDFrCT) is applied after quantization to the quantized image to obtain the original image as shown in Figure 1.

\subsection{Discrete Fractional Cosine Transform}

The advancement of DCT is DFrCT. Sequence of DCT $\{x[n], 0 \leq n \leq N-1)\}$ is defined [37-41] with equation (1) and (2);

$$
\begin{gathered}
x(k)=\alpha\left(k \sum_{n=0}^{N-1} x[n] \cos \left[\frac{2 n+1}{2 N}\right]\right), \text { for } 0 \leq k \leq N-1 \\
\text { where, } \alpha(k)=\left\{\begin{array}{lrr}
\frac{1}{\sqrt{N}} & \text { for } & k=0 \\
\frac{\sqrt{2}}{\sqrt{N}} & \text { for } & 1 \leq k \leq N-1
\end{array}\right\} \quad \text { (2) }
\end{gathered}
$$

Kernel matrix of 1D-DCT [42] is given by equation (3)

$$
E_{D C T}(k, n)=\left\{\begin{array}{cc}
\frac{1}{\sqrt{N}}, k=0 & 0 \leq n \leq N-1 \\
\sqrt{\frac{2}{N}} \cos \left[\frac{(2 n+1) \pi k}{2 N}\right] & 1 \leq k \leq N-1 ; 0 \leq n \leq N-1
\end{array}\right\}
$$

The inverse DCT is

$$
x[n]=\sum_{k=0}^{N-1} \alpha(k) X(k) \cos \left[\frac{(2 n+1) \pi k}{2 N}\right] ; 0 \leq n \leq N-1
$$

The kernel matrix of $\mathrm{N}$ point $\mathrm{DFrCT}[42-44]$ is

$$
\begin{aligned}
& C_{N, \alpha}=V^{N} D_{N}^{(2 \alpha / \pi)} V_{N}^{T} \\
& =V_{N}\left(\begin{array}{ccc}
1 & 0 & \\
e^{-2 j \alpha} & \ddots & \\
& & e^{-j 2(N-1) \alpha}
\end{array}\right)
\end{aligned}
$$

where $V_{N}=\left[V_{0}\left|V_{1}\right|----V_{2 N-2}\right]$, k the order DFT Hermite eigenvector is $V_{k}$.

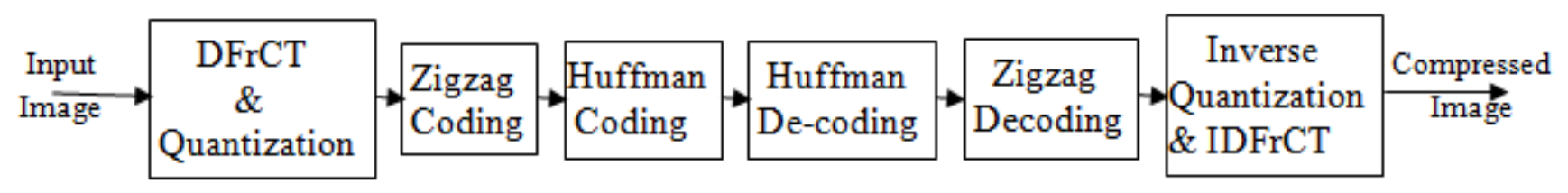

Fig. 1: Proposed Compression Model 
Unitarity, additivity of rotations, periodicity and reality [36] are the impoertant mathematical properties of DFrCT. 2D-DFrCT is used for encryption, decryption and image compression. In 2$\mathrm{D}$, angle of rotation $\alpha$ and $\beta$ are used separately.

\subsection{Zigzag Coding}

In zigzag coding data access from the low frequency components to high frequency components. Zigzag coding lead to long run of 0's and it converts the two dimensional array of an image into one dimensional array. In other words it converts the $\mathrm{M} \times \mathrm{N}$ matrix into $1 \mathrm{xN}$ matrix as shown in Figure 2, therefore zigzag coding play very vital role in image compression.

\subsection{Huffman Coding and Decoding}

Generally, the Huffman coding is used to eliminate the coding redundancy from the input image. It is a one form of statistical coding that minimize number of bits needs to represent the string of symbol. The main advantage of Huffman coding is to utilized the optimal code word that have least

average length. Its inverse process of coding is called decoding, by which original input matrix can be obtained easily.

\subsection{Zigzag Decoding}

In zigzag decoding reconstruction of original matrix from the one dimensional array. In other words, it convert one dimensional matrix into two dimensional matrix of an image.

\subsection{Quantization}

Quantization works efficiently to quantize all the coefficients obtained after applying DFrCT. The small coefficients obtained after applying DFrCT are quantized coarsely and the coefficients which are having large values are quantized to the nearest integer. After quantization, the coefficients are written on the compressed stream.

\section{Results and Discussion}

The most common used parameters to evaluate the image compression algorithms are PSNR and CR. From Equation (6), the PSNR value (in $\mathrm{dB}$ ) is used to compare the difference between the compressed image ' $o$ ' and the original input image ' $i$ '. In general, if obtained PSNR after compression is less means poor visual quality of image and for large PNSR means image visual quality is good, therefore for better image quality, the researcher aim is to get the larger PSNR value.

$$
\begin{aligned}
& M S E=\left[\frac{1}{255} \sum_{i=0}^{M-1} \sum_{j=0}^{N-1}[o(i, j)-i(i, j)]^{2}\right] \\
& P S N R=10 \log _{10}\left\lfloor\frac{M \times N}{M S E}\right\rfloor
\end{aligned}
$$

where $M \times N$ is the size of the image.

In order to evaluate the effectiveness and efficiency of proposed algorithm, four different test images have been used i.e,: "Lena", "Peppers", "Barbara" and "Baboon" as shown in Figure 3. It directly worked on the entire image without blocking or partitioning. Hence, this proposed algorithm performed well and provides compressed images which are free from blocking artifacts. This proposed technique provide good PSNR for all the images as compare to existing techniques due to which image quality has been increased after the compression without any blocking artifacts. 


\begin{tabular}{|l|l|l|l|l|l|l|l|}
\hline 69 & 52 & 47 & 47 & 46 & 57 & 67 & 81 \\
\hline 69 & 55 & 50 & 55 & 45 & 57 & 69 & 85 \\
\hline 66 & 57 & 55 & 48 & 47 & 58 & 70 & 86 \\
\hline 59 & 60 & 51 & 49 & 44 & 60 & 75 & 86 \\
\hline 59 & 58 & 50 & 47 & 48 & 63 & 51 & 96 \\
\hline 51 & 54 & 58 & 47 & 44 & 61 & 88 & 109 \\
\hline 57 & 54 & 65 & 41 & 46 & 58 & 74 & 116 \\
\hline 52 & 57 & 65 & 43 & 48 & 57 & 78 & 116 \\
\hline
\end{tabular}

\begin{tabular}{|c|c|c|c|c|c|c|c|}
\hline 30 & -1 & 0 & 0 & 0 & 0 & 0 & 0 \\
\hline-6 & 2 & 0 & 0 & 0 & 0 & 0 & 0 \\
\hline 0 & 0 & 0 & 0 & 0 & 0 & 0 & 0 \\
\hline-2 & 1 & 0 & 0 & 0 & 0 & 0 & 0 \\
\hline 0 & 0 & 0 & 0 & 0 & 0 & 0 & 0 \\
\hline 0 & 0 & 0 & 0 & 0 & 0 & 0 & 0 \\
\hline 0 & 0 & 0 & 0 & 0 & 0 & 0 & 0 \\
\hline 0 & 0 & 0 & 0 & 0 & 0 & 0 & 0 \\
\hline
\end{tabular}
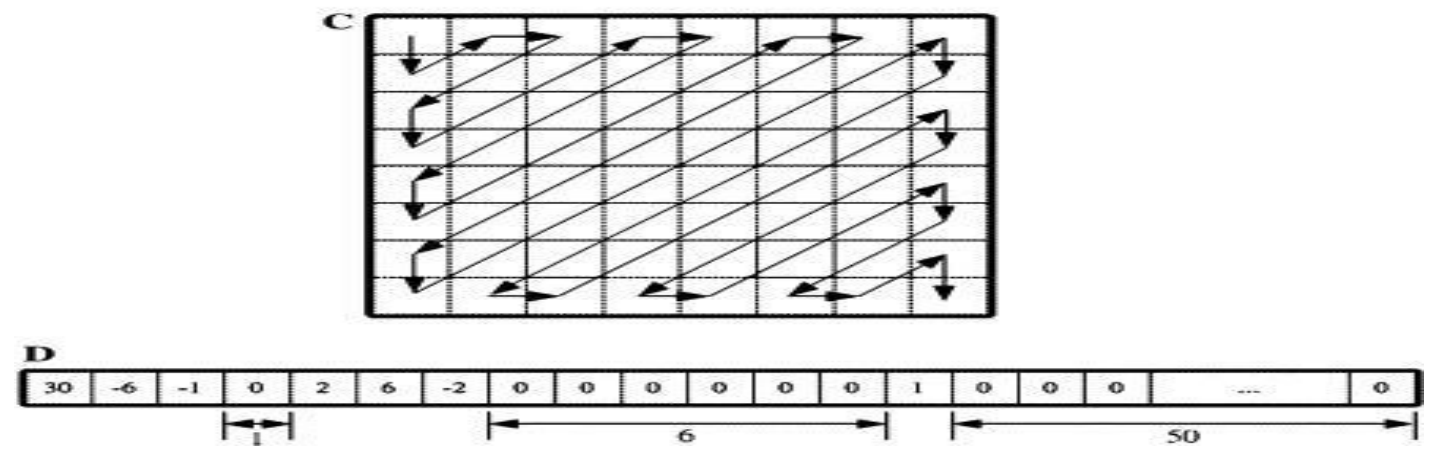

Fig. 2: An Example of DFrCT-Based Coding Technique. (A) An 8x8 Matrix(Subimage). (B) The Quantized DFrCT Coefficient Matrix of (A). (C) The Zig-Zag Scanning Pattern. (D) The Zig-Zag Scanned One Dimensional Matrix of (B)

As there is no possibility of optimization in other transforms based existing compression techniques. So, one more important advantage of this technique is that it uses DFrCT due to which optimization is possible by varying the fractional order ' $a$ '. Therefore, this proposed compression technique fulfilling the all the research gaps as mentioned earlier. Figure 4 gives the visual results of proposed algorithm for different images. Comparative results of various existing algorithms along with proposed algorithm are shown in Figure 5 and Table 1. From which we can conclude that proposed algorithm is superior in performance. Table 2 gives PSNR values for different fractional order ' $a$ ' for all compressed images. Finally, it is found that the best image compression is obtained by varying the fractional order ' $a$ ' ( 0 to 1$)$. For high PSNR values Table 2 and Figure 7 shows that optimum value of ' $a$ ' is 0.6 for Lena, Baboon and Barbara and 0.7 for Peppers. From Table 2 and Figure 7 we can conclude that the optimum value of fractional order ' $a$ ' is dependent on image. Table 3 and Figure 6 shows that comparative compression ratio for recently published algorithm and proposed algorithm, which depicts that proposed algorithm provide more compression ratio as compare to others. Less execution time and easy implementation are the attractive advantages of this proposed technique. Image compression with DFrCT works well in fractional domain and tries to save the bandwidth by varying fractional order 0 to 1 .



(a) Lena

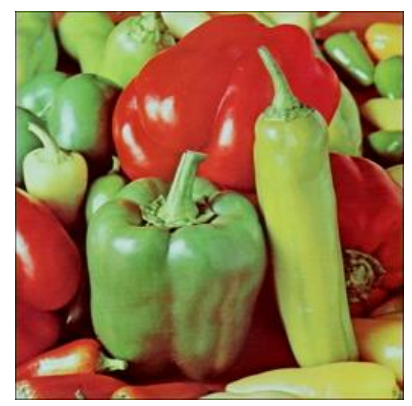

(b) Peppers

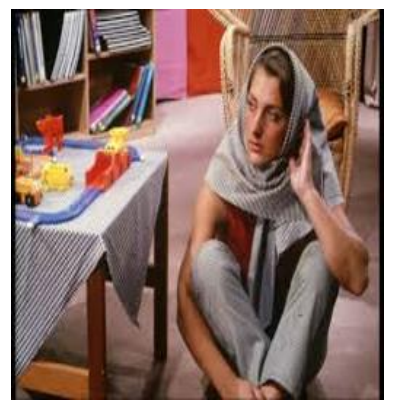

(c) Barbara

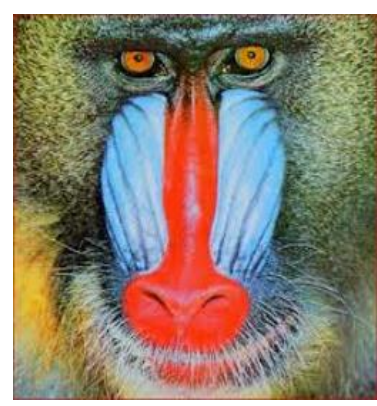

(d) Baboon

Fig. 3: Original Images 


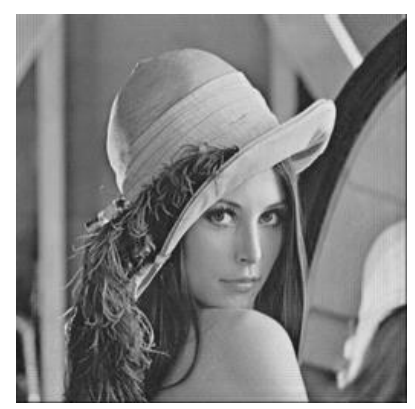

(a) Lena

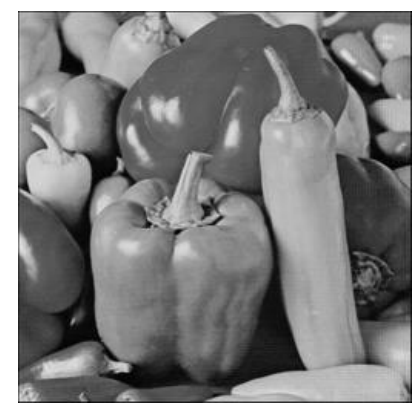

(b) Peppers



(c) Barbara

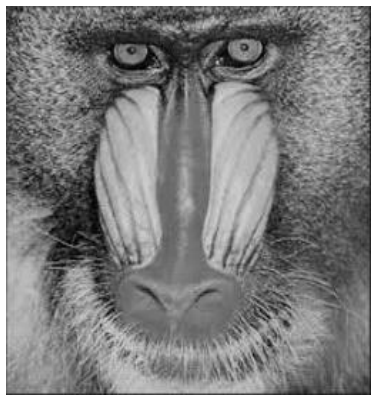

(d) Baboon

Fig. 4: Compressed Images By Proposed Technique



Fig. 5: Graph Shows the PSNR for Different Compression Techniques and Proposed Technique for Images (a)Lena, (b)Barbara, (c)Baboon and (d)Peppers.

Table 1. Comparison of Proposed Method with Different Methods

\begin{tabular}{|l|l|l|l|l|l|l|}
\hline \multirow{2}{*}{ Images } & \multicolumn{6}{|c|}{ PSNR (in dB) } \\
\cline { 2 - 7 } & JPEG & $\begin{array}{l}\text { Block Truncation } \\
\text { Coding (BTC) }\end{array}$ & $\begin{array}{l}\text { Singular Value } \\
\text { Decomposition } \\
\text { (SVD) }\end{array}$ & $\begin{array}{l}\text { Gaussian } \\
\text { Pyramid } \\
\text { (GP) }\end{array}$ & $\begin{array}{l}\text { C.S.Rawat } \\
{[\mathbf{1 5}]}\end{array}$ & $\begin{array}{l}\text { Proposed } \\
\text { Method }\end{array}$ \\
\hline Lena & 29.8870 & 29.6116 & 22.6225 & 15.6656 & 31.5739 & 33.0649 \\
\hline Barbara & 30.00 & 26.5894 & 20.4283 & 14.7322 & 32.7810 & 42.9752 \\
\hline Baboon & 31.3421 & 25.1743 & 20.0996 & 16.1080 & 35.8160 & 36.0058 \\
\hline Peppers & 34.2700 & 29.2346 & 22.3442 & 14.5351 & 39.2185 & 40.1281 \\
\hline
\end{tabular}

Table 2. PSNR for Different Fractional Order ' $a$ '

\begin{tabular}{|l|l|l|l|l|l|l|l|l|l|l|l|l|}
\hline \multirow{2}{*}{ Images } & \multicolumn{10}{|c|}{ PSNR (in dB) for different fractional order ' $a$ ' } \\
\cline { 2 - 15 } & $a=0$ & $a=0.1$ & $a=0.2$ & $a=0.3$ & $a=0.4$ & $a=0.5$ & $a=0.6$ & $a=0.7$ & $a=0.8$ & $a=0.9$ & $a=1$ \\
\hline Lena & 29.1933 & 30.1466 & 31.1514 & 31.4880 & 32.2296 & 32.3354 & $\mathbf{3 3 . 0 6 4 9}$ & 32.7057 & 32.0428 & 31.5458 & 30.7834 \\
\hline Barbara & 38.7292 & 40.1331 & 41.0359 & 41.5319 & 42.3117 & 42.3629 & $\mathbf{4 2 . 9 7 5 2}$ & 40.9277 & 38.9353 & 37.2686 & 34.4562 \\
\hline
\end{tabular}




\begin{tabular}{|l|l|l|l|l|l|l|l|l|l|l|l|l|}
\hline Baboon & & & & & & & & & & & \\
& 32.1813 & 33.0001 & 34.0066 & 34.3771 & 35.1626 & 35.2664 & $\mathbf{3 6 . 0 0 5 8}$ & 35.5677 & 34.8094 & 34.0582 & 32.2599 \\
\hline Peppers & & & & & & & & & & & & \\
& 34.0638 & 34.4808 & 35.5316 & 36.0736 & 36.9614 & 37.2041 & 38.1281 & $\mathbf{4 0 . 1 2 8 1}$ & 37.7744 & 36.5518 & 33.8705 \\
\hline
\end{tabular}

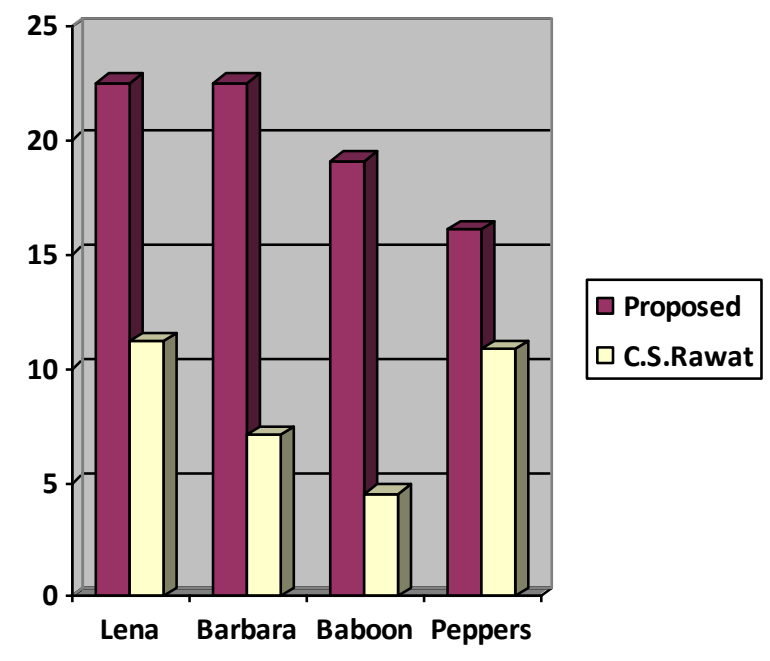

Table 3. Compression ratio for various images

\begin{tabular}{|l|l|l|}
\hline \multirow{2}{*}{ Images } & \multicolumn{2}{|l|}{ Compression Ratio } \\
\cline { 2 - 3 } & $\begin{array}{l}\text { C.S. Rawat } \\
{[\mathbf{1 5}]}\end{array}$ & $\begin{array}{l}\text { Proposed } \\
\text { Method }\end{array}$ \\
\hline Lena & 11.1544 & 22.5113 \\
\hline Barbara & 7.1266 & 22.4991 \\
\hline Baboon & 4.5253 & 19.0918 \\
\hline Peppers & 10.8559 & 16.0767 \\
\hline
\end{tabular}

Fig. 6: Graph Showing the Compression Ratio of C.S. Rawat [15] and Proposed Technique



(a) For Lena



(b) For Barbara 


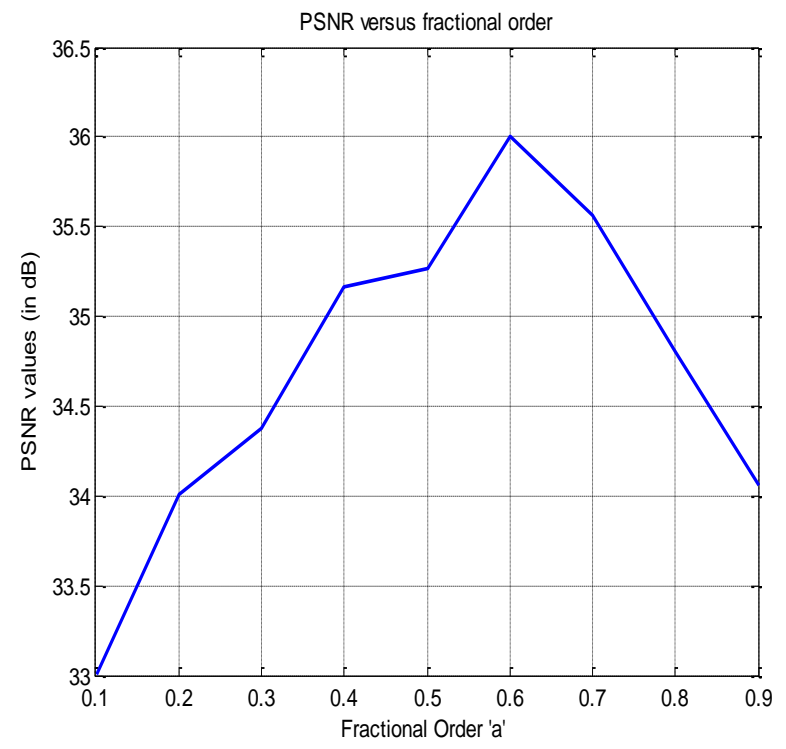

(c) For Baboon

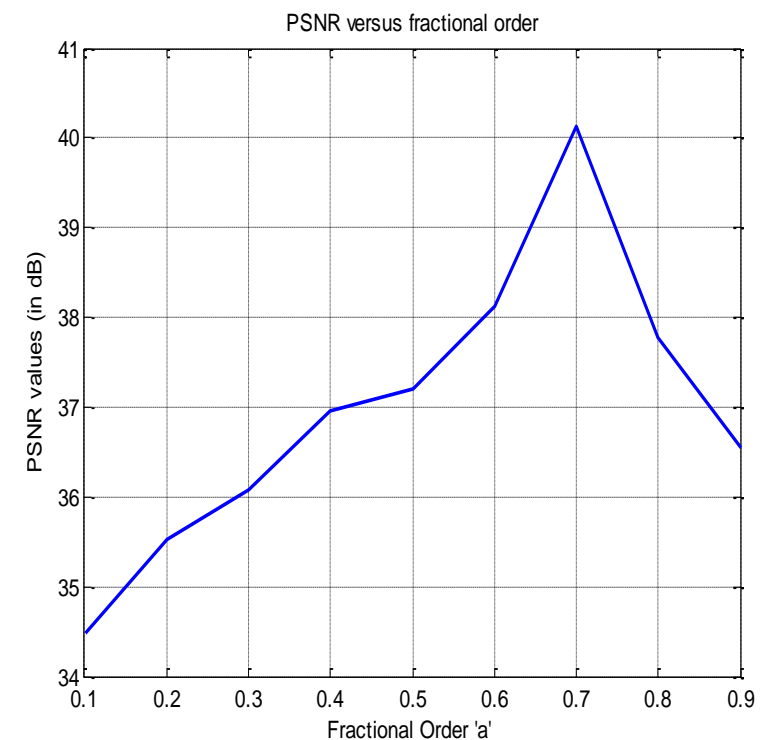

(d) For Peppers

Fig. 7: Different values of PSNR with varying fractional order ' $a$ ' $=0.1$ to 0.9 for (a) Lena, (b) Barbara, (c) Baboon and (d) Peppers

\section{Conclusion}

An efficient and novel technique for image compression in fractional domain has been proposed. The proposed technique efficiently and effectively compressed the images and optimum fractional order ' $a$ ' is computed using DFrCT. By working on blocks many existing techniques produces blocking artifacts but in this technique operation are not on nonoverlapped blocks or subimages. Therefore, blocking artifacts were repudiated by working on the entire image not by small blocks and this is the novelty of proposed technique. This proposed image compression model worked with single block (of size 256x256) which decreases the execution time. From the experimental and simulation results, it can be conclude that the proposed algorithm is robust and effective for compressing different type of images and provided very good compression and PSNR. The future scope will be the application of other fractional transform for image compression.

\section{References:}

[1] S. Annadurai and M. Sundaresan(2009). Wavelet Based Color Image Compression using Vector Quantization and Morphology. Proceedings of the International Conference on Advances in
Computing, Communication and Control., pp. 391-396.

[2] L. Krikor, S. Baba, T. Arif, and Z. Shaaban (2009). Image encryption using DCT and stream cipher. European Journal of Scientific Research. vol. 32 , no. 1,pp. $48-58$.

[3] A. Loussert, A. Alfalou, R. El-Sawda and A. Alkholidi (2008). Enhanced system for image's compression and encryption by addition of biometric characteristics. International Journal of Software Engineering and its Applications. vol. 2, no. 2,pp. 111-118.

[4] G. Ames. Image compression. International Journal of Advancements in Computing Technology. vol. 1, no. 2, (2002),pp. 205-220.

[5] N. Ahmed, T. Natarajan and K.R. Rao (1974). Discrete cosine transform. IEEE Trans. Comm., pp.90-93.

[6] Vivek Arya, P. Singh and K. Sekhon (2013). RGB image compression using two dimensional discrete cosine transform. International Journal of Engineering Trends and Technology. pp. 828832.

[7] Vivek Arya, P. Singh and K. Sekhon (2015). Medical image compression using two dimensional discrete cosine transform. 
International Journal of Electrical and Electronics Research. vol.3, issue 1, pp 156-164.

[8] Vivek Arya and J. Singh (2016). Robust image compression using two dimensional discrete cosine transform. International Journal of Electrical and Electronics Research. vol.4, issue 2, pp.187-192.

[9] K.R. Rao and P. Yip (1990). Discrete cosine transform: Algorithms advantages and applications. Academic Press, New York.

[10] P.M. Fanelle and A.K. Jainn (1986). Recursive block coding: A new approach to transform coding. IEEE Trans. Communication. pp. 161179.

[11] M. Bosi and G. Davidson (1992). High quality low bit rate audio transform coding for transmission and multimedia applications. Journal of Audio Eng. Soc. pp. 43-50.

[12] D. Solomaon, (2004). Data Compression: The complete Reference. Springer Verlag, Newyork.

[13] F. J. Macwilliams and N.J.A. Slone (1977). The Theory of Error Correcting Codes. Elsevier, Amsterdam.

[14] Y. T. Chan, (1995). Wavelet Basics. Kluwer Academic Publishers, Norwell, MA.

[15] C.S. Rawat and S. Meher (2013). A hybrid image compression scheme using DCT and fractal image compression. International Arab Journal of Information Technology. vol.10, no.6.

[16] N. Wiener (1929). Hermitian polynomials and Fourier analysis. J. Math. Phys., pp.70-73.

[17] E. U. Condon (1937). Immersion of the Fourier transform in a continuous group of functional transformations. Proc. Nat. Acad. Sci. USA, pp.158-164.

[18] H. Kober and Wurzeln aus der Hankel (1939). Fourier- und aus an-deren stetigen transformationen. Q. J. Math. Oxford Ser., pp. 45-49.

[19] B. Santhanam, J. H. McClellan (1996). The discrete rotational Fourier transform. IEEE Trans. Signal Processing, pp. 94-998.

[20] A.W. Lohmann (1993). Image rotation, Wigner rotation and the fractional Fourier transform. $J$. Opt. Soc. Am, pp. 2181-2186.

[21] V. Namias (1980). The fractional order Fourier transform and its application to quantum mechanics. J. Inst. Maths. Appl., pp. 241-265.

[22] L.B. Almeida (1994). The fractional Fourier transform and time frequency representations. IEEE Trans. Signal Process, pp. 3084-3091.
[23] C. Vijaya, J.S. Bhat (2006). Signal compression using discrete fractional Fourier transform and set partitioning in hierarchical tree. Signal Process, pp. 1976-1983.

[24] R.Tao, B. Deng, Y. Wang (2006). Research progress of the fractional Fourier transform in signal processing. Sci. China Ser. F Inf. Sci., pp. $1-25$.

[25] Bracewell, R. N (1986). The Fourier Transform and Its Applications. $2^{\text {nd }}$ Revised. McGraw Hill, New York.

[26] Abomhara, M., Zakaria, O., Khalifa, O.O (2010). An overview of video encryption techniques. Int. J. Comput. Theory Engineering, pp. 103-110.

[27] R. C. Gonzalez and R. E. Woods (2008). Digital Image Processing, 3rd edition.

[28] D. Mendlovic and H.M. Ozaktas (1993). Fractional Fourier transforms and their optical implementation. I. J. Opt. Soc. Am. A, pp. 18751881.

[29] N. M. Atakishiyev, L. E. Vicent and K.B. Wolf (1999). Continuous vs. discrete fractional Fourier transforms. J. Comput. Appl. Math. pp. 73-95.

[30] S.I. Yetik, M.A. Kutay and M.H. Ozaktas (2000). Continuous and discrete fractional Fourier domain decomposition. In IEEE Trans. pp. 93-96.

[31] M.H. Ozaktas, Z. Zalevsky and M.A. Kutay (2000). The Fractional Fourier Transform with Applications in Optics and Signal Processing. Wiley, New York.

[32] S.C. Pei, M.H. Yeh (2001). A novel method for discrete fractional Fourier transform computation. ISCAS, pp. 585-588.

[33] M.H. Yeh and S.C. Pei (2003). A method for the discrete-time fractional Fourier transform computation. Signal Process, pp. 1663-1669.

[34] S.C. Pei, M.H. Yeh, C.C. Tseng (1999). Discrete fractional Fourier transform based on orthogonal projections. IEEE Trans. Signal Processing., pp. 1335-1348.

[35] I. Djurovic, S. Stancovic and I. Pitas (2001). Digital watermarking in the fractional Fourier transformation domain. Journal of Netw. Comput. Applications. pp. 167-173.

[36] Rubio, J.G.V. and B. Santhanam (2005). On the multiangle centered discrete fractional Fourier transform. IEEE Signal Process. Letters.

[37] Gerek, O.N., Erden, M.F. (2000) The discrete fractional cosine transform. Proceedings of the 
IEEE Balkan Conference on Signal Processing, Communications, Circuits and Systems. Istanbul, Turkey.

[38] Lohmann, A.W., Mendlovic, D., Zalevsky, Z., Dorch, R.G. (1996), Some important fractional transformations for signal processing. Opt. Commun., pp. 18-20.

[39] Yip, P., Sine and Cosine transforms (1996). In Poularikas, A.D. (ed.) Transforms, The Handbook Applications. CRC Press, Alabama.

[40] Prudnikov, A.P., Brychkov, Y.A., Marichev, O.I. (1986), Integrals and Series VI: Elementary Functions. Gordon and Breech Science Publishers, New York.

[41] Ahmed, N., Natarajan, T., Rao, K.R. (1974), Discrete cosine transform. In IEEE Trans. Comp. C, pp.90-93.

[42] S.C. Pei, M.H Yeh, M.H. (2001). The discrete fractional cosine and sine transforms. IEEE Trans. Signal Process., pp.1198-1207.

[43] Narasimha, M.J., Peterson, A.M. (1978). On the computation of the discrete cosine transform. In IEEE Trans. Communication., pp.934-936.

[44] M.H. Yeh and S.C. Pei. (2003). A method for the discrete fractional Fourier transform computation. In IEEE Trans. Signal Processing. pp. 889-891.

[45] T. Zhao and Q. Ran (2019). The weighted fractional Fourier transform and its application in image encryption. Mathematical Problems in Engineering Hindawi. Vol. 2019, pp. 1-10.

[46] N. Kumar R., B.N. Jagadale and J.S. Bhat (2018), An improved image compression algorithm using wavelet and fractional cosine transform. International Journal of Image, Graphics and Signal Processing. vol.11, pp. 1927.

[47] Vivek Arya, \& Jugmendra Singh (2016). “Image Compression Algorithm Using Two Dimensional Discrete Cosine Transform" Published in Imperial Journal Interdisciplinary Research (IJIR), Vol-2, Issue-8.
Creative Commons Attribution License 4.0 (Attribution 4.0 International, CC BY 4.0)

This article is published under the terms of the Creative Commons Attribution License 4.0 https://creativecommons.org/licenses/by/4.0/deed.en US 